\title{
New evidence for a role of Bisphenol A in cell integrity. Implications in the human population
}

\author{
Rafael MORENO-GÓMEZ-TOLEDANO ${ }^{1, *}$; María I. ARENAS²; Esperanza VÉlEZ-VÉleZ ${ }^{3}$; Ricardo J. BOSCH \\ ${ }^{1}$ Universidad de Alcalá, Laboratory of Renal Physiology and Experimental Nephrology, Department of Biological Systems/Physiology, 28871, Alcalá de Henares, Spain \\ 2 Universidad de Alcalá, Cell Biology Unit, Department of Biomedicine and Biotechnology, 28871, Alcalá de Henares, Spain \\ 3 Jiménez Díaz Foundation, Fundación Jiménez Díaz School of Nursing, Madrid, 28040, Spain
}

Key words: Bisphenol A, Podocyte, Cytoskeleton, Cell Adhesion, Chronic Renal Diseases, Dialysis

\begin{abstract}
Bisphenol A (BPA) is a xenoestrogen known for its implications for the endocrine systems and several other organs, including the kidneys. Recent renal studies have shown that BPA can induce alterations of the cytoskeleton and cell adhesion mechanisms such as a podocytopathy with proteinuria and hypertension, alterations involved in the progression of renal diseases. These data and the fact that BPA is known to be present in the urine of almost the entire population strongly suggest the critical need to reevaluate BPA exposures considered safe.
\end{abstract}

\section{Introduction}

Bisphenol A (BPA) is a xenobiotic molecule classified within the category of endocrine disruptors, and thanks to its properties as an estrogen modulator, it is also called xenoestrogen (Taylor et al., 2011). BPA is found in countless everyday utensils, such as food containers, bottles, cans, toys, and is even found in clothing (Vandenberg et al., 2007; Dursun et al., 2016; EFSA, 2016; Li and Kannan, 2018; Freire et al., 2019). Its use is not restricted to the domestic sphere, as its ability to improve the qualities of plastics has made it an essential element in other industries, as it is used in the manufacture of cars, LED lights, and even medical-surgical material (Duty et al., 2013; Olabisi and Adewale, 2016; Testai et al., 2016).

\section{Presentation of the Viewpoint}

BPA is a molecule whose properties as an estrogen modulator were discovered in the 1930s by Dodds and Lawson (Dodds and Lawson, 1936). For this reason, numerous works study its possible relationship with reproductive or genitourinary disorders (Rochester, 2013; Ziv-Gal and Flaws, 2016; Pergialiotis et al., 2018; Tomza-Marciniak et al., 2018). However, in the last two decades, it has been shown that BPA can exert other types of actions on other organs or

*Address correspondence to: Rafael Moreno-Gómez-Toledano, rafael.moreno@uah.es

Received: 15 June 2021; Accepted: 08 July 2021 systems, favoring the accumulation of fatty tissue, the appearance of diabetes, cancer, and even cognitive and behavioral disorders (Provvisiero et al., 2016; Akash et al., 2020; Wu et al., 2020; Nesan et al., 2021). In recent years, our team has made interesting advances on the possible implications of BPA on the renal and vascular system.

Using experimental animals models we observed that BPA is capable to promote hypertension and renal damage (podocytopathy) as well as to participate in the mechanism of progression of chronic kidney disease (CKD) (OleaHerrero et al., 2014; Saura et al., 2014; Moreno-GómezToledano et al., 2020; Reventun et al., 2020), reviewed by Bosch et al. (2016).

Our first work with podocytes (cells that are part of the glomerular filtration barrier) were carried out with immortalized mouse cultures. Podocytes cell lines (mice and human) have a particular condition: at $33^{\circ} \mathrm{C}$, they remain undifferentiated, allowing their replication, and at $37^{\circ} \mathrm{C}$, they become quiescent, lose their mitotic capacity, and begin to differentiate for 11-15 days. After that time, the podocyte in culture is considered a differentiated, mature, and fully functional podocyte.

The administration of BPA to this cell line demonstrated that low exposure to this molecule (10 and $100 \mathrm{nM}$ ) could induce death mechanisms in cultured mouse podocytes (Olea-Herrero et al., 2014) (Fig. 1). Similarly, the intraperitoneal administration of BPA in mice demonstrated that BPA could induce cellular sampling mechanisms in the podocytes of the glomerulus of the animal (Olea-Herrero et al., 2014). These animals developed podocitopathy with 


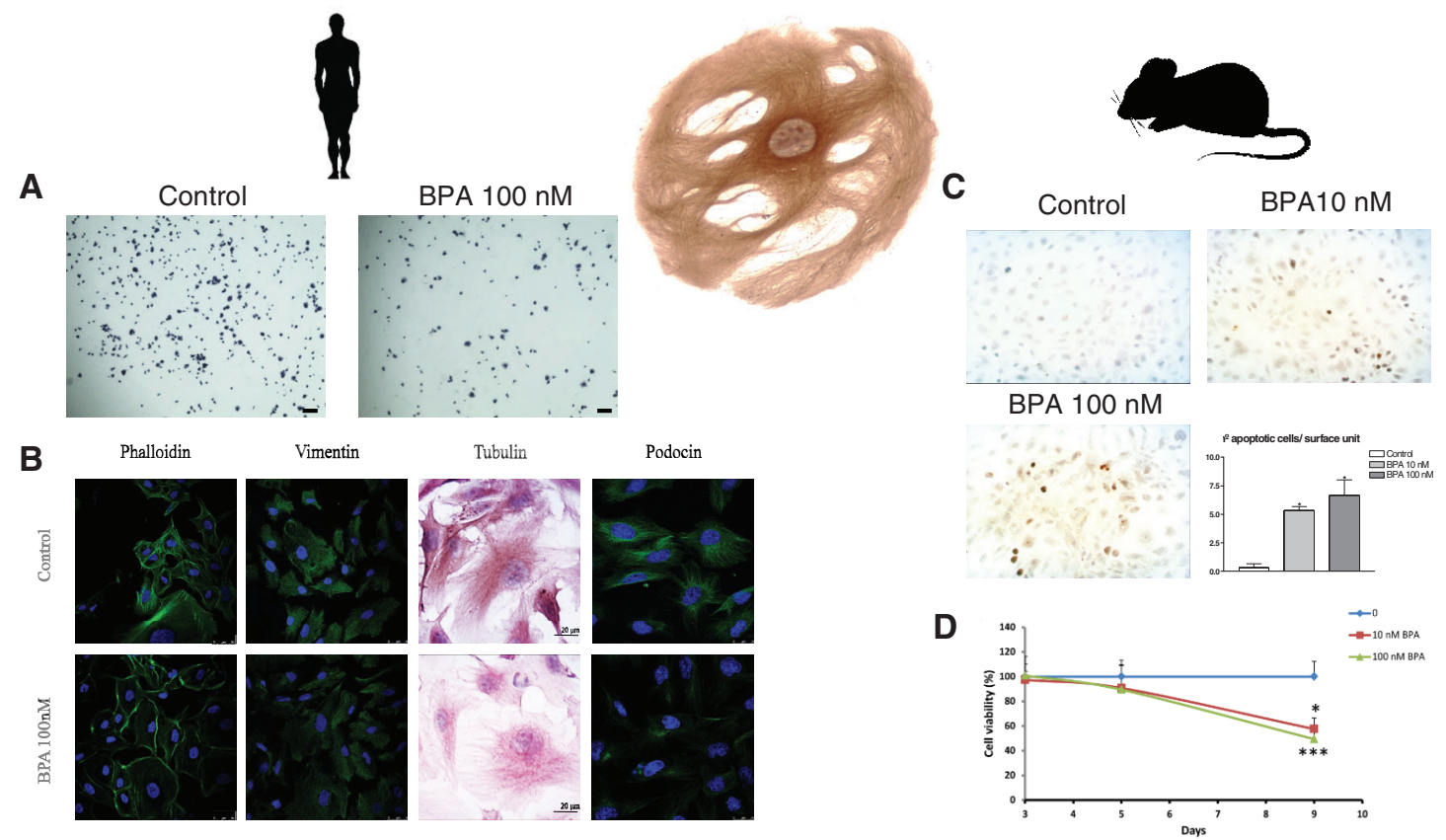

FIGURE 1. Interspecies differences in the effects promoted by BPA in the podocyte. A) Exposure to BPA induces a reduction in the adhesion capacity of the human podocyte $(\mathrm{x} 40)($ scale bar: $60 \mu \mathrm{m})$. B) Reduction in the relative expression of structural proteins after treatment with BPA, analyzed by immunofluorescence (scale bar: $50 \mu \mathrm{m}$ ) and immunohistochemistry (scale bar: $20 \mu \mathrm{m}$ ). C) In the mouse podocyte, the administration of BPA induces an increase in the mechanisms of cell death (x300). D) Treatment with BPA significantly reduces cell viability (MTT). Figure made with our own results published in the Journal of Cellular Physiology (Olea-Herrero et al., 2014), and Scientific Reports (Moreno-Gómez-Toledano et al., 2020).

proteinuria, similar to those seen in diabetic nephropathy. Although there are limitations when using mouse models for assessing renal failure or long-term histomorphological changes (Breyer et al., 2005), our findings may have pathophysiological implications since the amount of proteinuria and podocytes number are reliable predictors of the progression of renal disease (Meyer et al., 1999; D’Amico and Bazzi, 2003).

Subsequently, we observed that in human podocytes in culture (generously provided by Dr. M Saleem, University of Bristol), $100 \mathrm{nM}$ BPA promotes a novel type of podocytopathy characterized by an impairment of cell adhesion. Transcriptomic and proteomic studies demonstrated that BPA promotes alterations in the expression of structural and adhesion proteins (and messengers). Subsequent western blot and immunofluorescence assays demonstrated alterations in the relative expression of structural proteins such as actin, tubulin, vimentin, and podocin, as well as alterations in proteins related to adhesion mechanisms, such as cofilin-1, vinculin, E-cadherin, nephrin, VCAM-1, tenascin-C, and $\beta$-catenin (Moreno-Gómez-Toledano et al., 2020) (Fig. 1). In this way, it was possible to observe solid evidence that the cellular microenvironment and the elements that make it up, including xenobiotic compounds, can substantially affect the cellular structure.

Adamakis et al. (2018) observed that BPA in the aquatic environment, at environmentally relevant BPA concentration, was capable of promoting alterations in the cytoskeleton of the seagrass Cymodocea nodosa, even stating that the integrity of the actin filament is the most sensitive biomarker to exposure to BPA. Stavropoulou et al. (2018) also observed BPAmediated alterations in the actin filaments of the Zea mays (corn) plant. In cell cultures, Yin et al. (2020) described BPA-mediated cytoskeletal alterations in a mouse neuroblastoma cell line (neuro-2a cells). When using BPA doses in the micromolar range, they observed a reduction in the number of dendrites and a lower signal intensity when performing phalloidin (F-actin) immunofluorescence. Similarly, Rameshrad et al. (2018) observed that BPA is capable of inducing alterations in the expression of VCAM (adhesion protein) in human umbilical vein endothelial cells, also in the micromolar range.

\section{Analysis of the Impact of the Viewpoint}

It is evident that BPA can affect cell structure, not only in human cell cultures but also in murine cell cultures and even aquatic and terrestrial plants. This effect in the podocyte is especially interesting since its inability to regenerate makes it a cell of particular relevance in $\mathrm{CKD}$ (Glassock and Rule, 2016). Technically, BPA-induced podocyte loss could be as damaging to kidney function as cell death. The loss of podocytes induces an increase in the compensatory mesangial matrix, progressively reducing the renal filtration capacity. However, new studies have observed that although the podocyte does not have replicative capacity per se, the surrounding glomerular parietal epithelial cells could play a decisive role in podocyte regeneration, differentiating towards this cell type (Shankland et al., 2017). In any case, when the regenerative capacity cannot compensate for the loss, the kidney will progress to CKD. Since BPA is a possible environmental factor involved in this type of pathology, the degree of exposure to which the susceptible population is exposed, 
such as patients in intensive care or patients undergoing dialysis techniques, is of particular importance.

As we previously described, mean urinary BPA values have been observed in patients undergoing conventional dialysis between $52.73-155.84 \mathrm{ng} / \mathrm{ml}(1.11-3.28 \mu \mathrm{g} / \mathrm{kg}$ BW/day, 230.98-682.64 nM) (Moreno-Gómez-Toledano et al., 2021). These values are lower than those considered safe by the European Food Safety Authority, $4 \mu \mathrm{g} / \mathrm{kg}$ BW/day (TDI, Tolerable Daily Intake) (EFSA, 2016). However, this dose has been calculated using animal models as reference (Tyl et al., 2008), to which several correction factors were applied. As shown in Fig. 1, our cellular models show considerable evidence of the effects produced by BPA at the same doses, which should be a critical element to take into account when extrapolating animal studies to humans. Furthermore, the doses observed in CKD patients in dialysis treatment are lower than the proposed TDI but between 2 and 6 times higher than the concentrations used in the cell models.

However, it must be mentioned that BPA is among other chemicals that can be found circulating in the body. In recent years, various compounds have been identified, such as phthalates (Wang et al., 2019), as well as other phenolic derivatives, such as bisphenol S, F, or AF (BPS, BPF, or BPAF, respectively) (Chen et al., 2016). It has been observed that the combination of these elements could enhance the damage that they can already exert individually. Thus, it has been observed that the combination of BPA and dibutyl phthalate (DBP) increases cytotoxicity, oxidative stress, and genotoxicity in liver cell cultures (Li et al., 2017). In animal models, it has been determined that co-exposure of BPA with Di-(2-Ethylhexyl) -phthalate (DEHP) appears to increase susceptibility to tumor development (Zhang et al., 2021).

Furthermore, computer models that have recently been published showed synergy or antagonism as a function of the combination of phenolic derivatives (Jatkowska et al., 2021). According to Kataria et al. (2015), oxidative stress might represent a common pathway that mediates renal injury associated with exposure to environmental chemicals such as BPA, phthalates, polycyclic aromatic hydrocarbon, polychlorinated biphenyl, perfluoroalkyl acid as well as dioxins. This mechanism has biological plausibility and justifies further investigation when examining the adverse effects of these chemicals. Interestingly, these authors also suggest that other functional disturbances contribute to the adverse cardiorenal effects elicited by the described compounds, including effects on modifiable patientassociated factors, such as obesity.

\section{Conclusion}

The latest advances in BPA study have determined that it is a molecule with the potential to induce alterations in the cytoskeleton and the capacity for cell adhesion. The concentrations to which the susceptible population is exposed could worsen their pathologies, particularly in patients with CKD. The widespread usage of BPA, especially in the composition of the surgical medical material, should be evaluated and act accordingly, as it could be a crucial factor in the evolution of specific pathologies. Future translational studies need to evaluate the impact of BPA in the human population and reevaluate BPA exposures considered safe.

Copyright of Figures: We want to thank the Journal of Cellular Physiology (Olea-Herrero et al., 2014), and Scientific Reports (Moreno-Gómez-Toledano et al., 2020) for publishing our research results, part of which has been used in Fig. 1.

Author Contribution: The authors confirm contribution to the paper as follows: study conception and design: R. MorenoGómez-Toledano; M. I. Arenas; E. Vélez-Vélez; R. J. Bosch; draft manuscript preparation: R. Moreno-Gómez-Toledano; M. I. Arenas; E. Vélez-Vélez; R. J. Bosch. All authors reviewed the results and approved the final version of the manuscript.

Funding Statement: This work was supported in part by grants from Instituto de Salud Carlos III (PI15/02139) -Fondo Europeo de Desarrollo Regional (FEDER)-. R. Moreno-Gómez-Toledano is recipient of a research contract from CAM (B2017-BMD-3686).

Conflicts of Interest: The authors declare that they have no conflicts of interest to report regarding the present study.

\section{References}

Adamakis IDS, Malea P, Panteris E (2018). The effects of Bisphenol A on the seagrass Cymodocea nodosa: Leaf elongation impairment and cytoskeleton disturbance. Ecotoxicology and Environmental Safety 157: 431-440.

Akash M, Sabir S, Rehman K (2020). Bisphenol A-induced metabolic disorders: From exposure to mechanism of action. Environmental Toxicology and Pharmacology 77: 103373. DOI 10.1016/j.etap.2020.103373.

Bosch RJ, Quiroga B, Munoz-Moreno C, Olea-Herrero N, Arenas MI et al. (2016). Bisphenol A: An environmental factor implicated in renal vascular damage. Nefrología (English Edition) 36: 5-9.

Breyer MD, Böttinger E, Brosius FC, Coffman TM, Harris RC et al. (2005). Mouse Models of Diabetic Nephropathy. Journal of the American Society of Nephrology 16: 27-45.

Chen D, Kannan K, Tan H, Zheng Z, Feng YLL, Wu Y, Widelka M (2016). Bisphenol Analogues Other Than BPA: Environmental Occurrence, Human Exposure, and Toxicity-A Review. Environmental Science \& Technology 50: 5438-5453.

D’Amico G, Bazzi C (2003). Pathophysiology of proteinuria. Kidney International 63: 809-825.

Dodds EC, Lawson W (1936). Synthetic Estrogenic agents without phenanthrene nucleus. Nature 137: 996.

Dursun E, Fron-Chabouis HH, Attal JP, Raskin A (2016). Bisphenol a release: Survey of the composition of dental composite resins. Open Dentistry Journal 10: 446-453.

Duty SM, Mendonca K, Hauser R, Calafat AM, Ye X et al. (2013). Potential sources of bisphenol $\mathrm{A}$ in the neonatal intensive care unit. Pediatrics 131: 483-489.

EFSA (2016). Scientific Opinion on the risks to public health related to the presence of bisphenol A (BPA) in foodstuffs. EFSA Journal 13: 3978.

Freire C, Molina-Molina JM, Iribarne-Durán LM, Jiménez-Díaz I, Vela-Soria $\mathrm{F}$ et al. (2019). Concentrations of bisphenol A and parabens in socks for infants and young children in 
Spain and their hormone-like activities. Environment International 127: 592-600.

Glassock RJ, Rule AD (2016). Aging and the kidneys: Anatomy, physiology and consequences for defining chronic kidney disease. Nephron 134: 25-29.

Jatkowska N, Kudłak B, Lewandowska P, Liu W, Williams MJ, Schiöth HB (2021). Identification of synergistic and antagonistic actions of environmental pollutants: Bisphenols $\mathrm{A}, \mathrm{S}$ and $\mathrm{F}$ in the presence of DEP, DBP, BADGE and BADGE-2HCl in three component mixtures. Science of the Total Environment 767: 144286.

Kataria A, Trasande L, Trachtman H (2015). The effects of environmental chemicals on renal function. Nature Reviews Nephrology 11: 610-625.

Li AJ, Kannan K (2018). Elevated concentrations of bisphenols, benzophenones, and antimicrobials in pantyhose collected from six countries. Environmental Science \& Technology 52: 10812-10819.

Li X, Yin P, Zhao L (2017). Effects of individual and combined toxicity of bisphenol A, dibutyl phthalate and cadmium on oxidative stress and genotoxicity in HepG 2 cells. Food and Chemical Toxicology 105: 73-81.

Meyer TW, Bennett PH, Nelson RG (1999). Podocyte number predicts long-term urinary albumin excretion in Pima Indians with Type II diabetes and microalbuminuria. Diabetologia 42: 1341-1344.

Moreno-Gómez-Toledano R, Arenas MI, González-Martínez C, Olea-Herrero N, Reventún P et al. (2020). Bisphenol A impaired cell adhesion by altering the expression of adhesion and cytoskeleton proteins on human podocytes. Scientific Reports 10: 16638.

Moreno-Gómez-Toledano R, Arenas MI, Sánchez-Esteban S, Cook A, Saura $M$ et al. (2021). Critical analysis of human exposure to bisphenol a and its novel implications on renal, cardiovascular and hypertensive diseases. Hot Topics in Endocrinology and Metabolism [Working Title], pp. 1-20. IntechOpen.

Nesan D, Feighan KM, Antle MC, Kurrasch DM (2021). Gestational low-dose BPA exposure impacts suprachiasmatic nucleus neurogenesis and circadian activity with transgenerational effects. Science Advances 7: eabd1159.

Olabisi O, Adewale K (2016). Handbook of thermoplastics, second edition. Plastics Engineering 41: 1003.

Olea-Herrero N, Arenas MI, Muñóz-Moreno C, Moreno-GómezToledano R, González-Santander M et al. (2014). Bisphenol-A induces podocytopathy with proteinuria in mice. Journal of Cellular Physiology 229: 2057-2066.

Pergialiotis V, Kotrogianni P, Christopoulos-Timogiannakis E, Koutaki D, Daskalakis G et al. (2018). Bisphenol A and adverse pregnancy outcomes: A systematic review of the literature. Journal of Maternal-Fetal \& Neonatal Medicine 31: $3320-3327$.

Provvisiero DP, Pivonello C, Muscogiuri G, Negri M, de Angelis C, Simeoli C, Pivonello R, Colao A (2016). Influence of bisphenol A on type 2 diabetes mellitus. International Journal of Environmental Research and Public Health 13: 989. DOI 10.3390/ijerph13100989.

Rameshrad M, Imenshahidi M, Razavi BM, Iranshahi M, Hosseinzadeh H (2018). Bisphenol A vascular toxicity:
Protective effect of Vitis vinifera (grape) seed extract and resveratrol. Phytotherapy Research 32: 2396-2407.

Reventun P, Sanchez-Esteban S, Cook A, Cuadrado I, Roza C, Moreno-Gomez-Toledano R, Muñoz C, Zaragoza C, Bosch RJ, Saura M (2020). Bisphenol A induces coronary endothelial cell necroptosis by activating RIP3/CamKII dependent pathway. Scientific Reports 10: 4190. DOI 10.1038/s41598-020-61014-1.

Rochester JR (2013). Bisphenol A and human health: A review of the literature. Reproductive Toxicology 42: 132-155.

Saura M, Marquez S, Reventun P, Olea-Herrero N, Arenas MI et al. (2014). Oral administration of bisphenol A induces high blood pressure through angiotensin II/CaMKII-dependent uncoupling of eNOS. FASEB Journal 28: 4719-4728.

Shankland SJ, Freedman BS, Pippin JW (2017). Can podocytes be regenerated in adults? Current Opinion in Nephrology and Hypertension 26: 154-164.

Stavropoulou K, Adamakis IDS, Panteris E, Arseni EM, Eleftheriou EP (2018). Disruption of actin filaments in Zea mays by bisphenol A depends on their crosstalk with microtubules. Chemosphere 195: 653-665.

Taylor JA, Richter CA, Ruhlen RL, vom Saal FS, Saal FS vom (2011). Estrogenic environmental chemicals and drugs: Mechanisms for effects on the developing male urogenital system. Journal of Steroid Biochemistry and Molecular Biology 127: 83-95.

Testai E, Hartemann P, Rodriguez-Farre E, Rastogi SC, Bustos J et al. (2016). The safety of the use of bisphenol A in medical devices. Regulatory Toxicology and Pharmacology 79: 106-107.

Tomza-Marciniak A, Stępkowska P, Kuba J, Pilarczyk B (2018). Effect of bisphenol A on reproductive processes: A review of in vitro, in vivo and epidemiological studies. Journal of Applied Toxicology 38: 51-80.

Tyl RW, Myers CB, Marr MC, Sloan CS, Castillo NP et al. (2008). Two-generation reproductive toxicity study of dietary bisphenol A in CD-1 (Swiss) mice. Toxicological Sciences: An Official Journal of the Society of Toxicology 104: 362-384.

Vandenberg LN, Hauser R, Marcus M, Olea N, Welshons WV (2007). Human exposure to bisphenol A (BPA). Reproductive Toxicology 24: 139-177.

Wang Y, Zhu H, Kannan K (2019). A review of biomonitoring of phthalate exposures. Toxics 7: 21.

Wu W, Li M, Liu A, Wu C, Li D, Deng Q, Zhang B, Du J, Gao X, Hong Y (2020). Bisphenol A and the risk of obesity a systematic review with meta-analysis of the epidemiological evidence. Dose-Response 18: 1559325820916949. DOI $10.1177 / 1559325820916949$.

Yin Z, Hua L, Chen L, Hu D, Li J, An Z, Tian T, Ning H, Ge Y (2020). Bisphenol-A exposure induced neurotoxicity and associated with synapse and cytoskeleton in Neuro-2a cells. Toxicology in Vitro 67: 104911. DOI 10.1016/j.tiv.2020.104911.

Zhang X, Cheng C, Zhang G, Xiao M, Li L, Wu S, Lu X (2021). Coexposure to BPA and DEHP enhances susceptibility of mammary tumors via up-regulating Esr1/HDAC6 pathway in female rats. Ecotoxicology and Environmental Safety 221: 112453.

Ziv-Gal A, Flaws JA (2016). Evidence for bisphenol A-induced female infertility: A review (2007-2016). Fertility and Sterility 106: 827-856. 